\section{PSQ-027 OUTPATIENT PARENTERAL ANTIMICROBIAL THERAPY WORKING GROUP IN A HOSPITAL AT HOME UNIT: THE ESSENTIAL ROLE OF PHARMACISTS}

A Simões* ${ }^{*}$ A Brito, A Alcobia. Hospital Garcia De Orta, Pharmacy, Almada, Portugal

10.1136/ejhpharm-2020-eahpconf.344

Background and importance Outpatient parenteral antimicrobial therapy (OPAT) has significantly increased since the implementation in 2015 of a hospital at home (HAH) unit. This increase was largely due to the versatility of once daily OPAT administration, advances in vascular access and infusion devices, and high acceptance by patients and healthcare professionals. The implementation also decreased cost, and improved safety and efficacy in a large number of infectious diseases. ${ }^{1}$ In 2018 , an OPAT working group at a $\mathrm{HaH}$ unit was formed to optimise intravenous antimicrobial (IA) therapy, developing therapeutic protocols, and improving OPAT administration procedures at the patient's home.

Aim and objectives To assess the importance of integrating a pharmacist into the HaH OPAT working group to optimise parenteral antimicrobial therapy.

Material and methods A bibliographic review and analysis of summary of products characteristics of IA therapy in the hospital was carried out to evaluate the properties, dosage, dose, administration routes and stability after reconstitution and/or dilution. Assessment of patient profiles treated with OPAT at the $\mathrm{HaH}$ during the first semester of 2017 was done and identification of the main differences compared with patients admitted to the conventional medicine service who refused to be admitted to the $\mathrm{HaH}$ during the same period.

Results The literature review allowed the development of a summary table with the most relevant information: reconstitution, dilution, stability, administration routes, incompatibilities, interactions and alerts. In April 2018, $\mathrm{HaH}$ therapeutic protocols were implemented according to IA selection and administration routes, as well as the use of programmable infusion devices that allow continuous or intermittent infusion according to the stability of each IA.

An assessment was made 6 months after the implementation of these measures, demonstrating that the use of third generation cephalosporins were successfully substituted with second generation cephalosporins in 30\% of patients.

Conclusion and relevance The literature review contributed towards optimising the selection and use of IA, promoting its rational use, a fact proven by the decrease in third generation cephalosporin use. Study of the routes of administration and stability after reconstitution and/or dilution allowed minimisation of adverse effects. Therefore, the integration of a pharmacist into the $\mathrm{HaH}$ OPAT working group contributed towards increasing the effectiveness of OPAT and patient safety.

\section{REFERENCES AND/OR ACKNOWLEDGEMENTS}

1. Chung $E$, et al. Development and implementation of a pharmacist-managed OPAT program. Scholarship and Professional Work 2016:229.

No conflict of interest.

\section{PSQ-028 PRESCRIPTION PROFILE OF ISAVUCONAZOLE IN THE REAL WORLD CLINICAL PRACTICE}

${ }^{1} \mathrm{~S}$ Guijarro Herrera*, 'E Gonzalez Del Valle, ${ }^{1} \mathrm{R}$ Garcia Fumero, ${ }^{1} \mathrm{~S}$ Cano Dominguez, ${ }^{2} \mathrm{M}$ Rodriguez Goicochea, ${ }^{1} \mathrm{~N}$ Márquez Pete. ${ }^{1}$ Hospital Universitario Virgen De Las Nieves, Hospital Pharmacy, Granada, Spain; ${ }^{2}$ Hospital Santa Caterina, Hospital Pharmacy, Girona, Spain

\subsection{6/ejhpharm-2020-eahpconf.345}

Background and importance Early antifungal therapy for invasive pulmonary aspergillosis has been associated with better survival outcomes in immunocompromised patients. Isavuconazole, as well as caspofungin and liposomal amphotericin B $(\mathrm{LAmB})$, is a recommended alternative to voriconazole when contraindicated due to its toxicity or pharmacokinetic profile.

Aim and objectives To assess the efficacy and safety of isavuconazole therapy in patients diagnosed with invasive fungal disease (IFD) in clinical practice.

Material and methods A retrospective observational study was performed in a third level hospital. Patients treated with isavuconazole between December 2017 and October 2019 were included. Demographic, clinical and therapeutic variables were collected. Diagnostic criteria of IFD were assessed in accordance with the European Organisation for Research and Treatment of Cancer/Infectious Diseases Mycoses Study Group (EORCT/MSG). These data were obtained from electronic medical records.

Results Fifteen patients were recruited: 64\% men, mean age $50 \pm 17$ years. Median days of treatment was 47 (IQR 47142). Doses were prescribed following the drug's label. The main diagnosis was haematological malignancy (73.3\%), 81.8\% of which had undergone haematopoietic stem cell transplantation. IFDs included: proven $(n=0)$, probable $(n=6)$ and possible $(n=4)$ aspergillosis; and possible mucormycosis $(n=1)$. One patient was diagnosed with aspergillus vertebral osteomyelitis. Overall, $53.3 \%$ of patients had previously been treated with voriconazole, $20 \%$ with LAmB, $20 \%$ were treatment naïve and one patient was treated with posaconazole for mucormycosis. Reasons for drug switching were: to avoid potential drug interactions (40\%); voriconazole related adverse effects (33\%); LAmB toxicity in one patient; ineffectiveness in one patient; and isavuconazole's better safety profile in another patient. However, hepatobiliary adverse effects were reported in $26.6 \%$ of patients and ocular toxicity in $6.7 \%$. Regarding the efficacy of isavuconazole, $26.6 \%$ of patients died during treatment and only two patients were considered cured. At the time of data cut-off, only one patient was continuing treatment.

Conclusion and relevance Isavuconazole appears to be an emerging therapy for IFDs in our hospital. Its better pharmacokinetic profile and tolerance means a therapeutic option for complicated patients. Nevertheless, the poor prognosis hinders efficacy assessment in this setting and hence a more cost effectiveness selection among the available antifungals should be performed.

\section{REFERENCES AND/OR ACKNOWLEDGEMENTS}

No conflict of interest. 